ELECTRONIC RESEARCH ANNOUNCEMENTS OF THE AMERICAN MATHEMATICAL SOCIETY

Volume 7, Pages 17-27 (April 18, 2001)

S $1079-6762(01) 00090-7$

\title{
A STRETCHED EXPONENTIAL BOUND ON THE RATE OF GROWTH OF THE NUMBER OF PERIODIC POINTS FOR PREVALENT DIFFEOMORPHISMS I
}

\author{
VADIM YU. KALOSHIN AND BRIAN R. HUNT \\ (Communicated by Svetlana Katok)
}

\begin{abstract}
For diffeomorphisms of smooth compact manifolds, we consider the problem of how fast the number of periodic points with period $n$ grows as a function of $n$. In many familiar cases (e.g., Anosov systems) the growth is exponential, but arbitrarily fast growth is possible; in fact, the first author has shown that arbitrarily fast growth is topologically (Baire) generic for $C^{2}$ or smoother diffeomorphisms. In the present work we show that, by contrast, for a measure-theoretic notion of genericity we call "prevalence", the growth is not much faster than exponential. Specifically, we show that for each $\delta>0$, there is a prevalent set of ( $C^{1+\rho}$ or smoother) diffeomorphisms for which the number of period $n$ points is bounded above by $\exp \left(\mathrm{Cn}^{1+\delta}\right)$ for some $C$ independent of $n$. We also obtain a related bound on the decay of the hyperbolicity of the periodic points as a function of $n$. The contrast between topologically generic and measure-theoretically generic behavior for the growth of the number of periodic points and the decay of their hyperbolicity shows this to be a subtle and complex phenomenon, reminiscent of KAM theory.
\end{abstract}

\section{INTRODUCTION}

Let $\operatorname{Diff}^{r}(M)$ be the space of $C^{r}$ diffeomorphisms of a finite-dimensional smooth compact manifold $M$ with the uniform $C^{r}$-topology, where $\operatorname{dim} M \geq 2$, and let $f \in \operatorname{Diff}^{r}(M)$. Consider the number of periodic points of period $n$,

$$
P_{n}(f)=\#\left\{x \in M: x=f^{n}(x)\right\} .
$$

The main question of this paper is:

Question 1. How quickly can $P_{n}(f)$ grow with $n$ for a "generic" $C^{r}$ diffeomorphism $f$ ?

We put the word "generic" in quotes because, as the reader will see, the answer depends on the notion of genericity.

For technical reasons one sometimes counts only isolated points of period $n$; let

$$
\begin{aligned}
P_{n}^{i}(f)=\#\left\{x \in M: x=f^{n}(x) \text { and } y\right. & \neq f^{n}(y) \\
& \text { for } y \neq x \text { in some neighborhood of } x\} .
\end{aligned}
$$

Received by the editors December 21, 2000.

2000 Mathematics Subject Classification. Primary 37C20, 37C27, 37C35, 34C25, 34C27.

Key words and phrases. Periodic points, prevalence, diffeomorphisms.

(C)2001 American Mathematical Society 
We call a diffeomorphism $f \in \operatorname{Diff}^{r}(M)$ an Artin-Mazur diffeomorphism (or simply $A-M$ diffeomorphism) if the number of isolated periodic orbits of $f$ grows at most exponentially fast, i.e., for some number $C>0$,

$$
P_{n}^{i}(f) \leq \exp (C n) \text { for all } n \in \mathbb{Z}_{+} .
$$

Artin and Mazur $[\mathrm{AM}$. proved the following result.

Theorem 1.1. For $0 \leq r \leq \infty, A$ - $M$ diffeomorphisms are dense in $\operatorname{Diff}^{r}(M)$ with the uniform $C^{r}$-topology.

We say that a point $x \in M$ of period $n$ for $f$ is hyperbolic if $d f^{n}(x)$, the derivative of $f^{n}$ at $x$, has no eigenvalues with modulus 1 . (Notice that a hyperbolic solution to $f^{n}(x)=x$ must also be isolated.) We call $f \in \operatorname{Diff}^{r}(M)$ a strongly Artin-Mazur diffeomorphism if for some number $C>0$,

$$
P_{n}(f) \leq \exp (C n) \text { for all } n \in \mathbb{Z}_{+},
$$

and all periodic points of $f$ are hyperbolic (whence $P_{n}(f)=P_{n}^{i}(f)$ ). In [K1] an elementary proof of the following extension of the Artin-Mazur result is given.

Theorem 1.2. For $0 \leq r<\infty$, strongly $A$-M diffeomorphisms are dense in $\operatorname{Diff}^{r}(M)$ with the uniform $C^{r}$-topology.

According to the standard terminology, a set in $\operatorname{Diff}^{r}(M)$ is called residual if it contains a countable intersection of open dense sets, and a property is called (Baire) generic if diffeomorphisms with that property form a residual set. It turns out the A-M property is not generic, as is shown in [K2]. Moreover:

Theorem 1.3 ([K2] $)$. For any $2 \leq r<\infty$ there is an open set $\mathcal{N} \subset \operatorname{Diff}^{r}(M)$ such that for any given sequence $a=\left\{a_{n}\right\}_{n \in \mathbb{Z}_{+}}$there is a Baire generic set $\mathcal{R}_{a}$ in $\mathcal{N}$ depending on the sequence $a_{n}$ with the property that if $f \in \mathcal{R}_{a}$, then for infinitely many $n_{k} \in \mathbb{Z}_{+}$we have $P_{n_{k}}^{i}(f)>a_{n_{k}}$.

Of course, since $P_{n}(f) \geq P_{n}^{i}(f)$, the same statement can be made about $P_{n}(f)$. But in fact it is shown in [K2] that $P_{n}(f)$ is infinite for $n$ sufficiently large, due to a continuum of periodic points, for at least a dense set of $f \in \mathcal{N}$.

The proof of this theorem is based on a result of Gonchenko-Shilnikov-Turaev GST1. Two slightly different detailed proofs of their result are given in K2 and GST2. The proof in [K2 relies on a strategy outlined in GST1.

However, it seems unnatural that if you pick a diffeomorphism at random then it may have an arbitrarily fast growth of the number of periodic points. Moreover, Baire generic sets in Euclidean spaces can have zero Lebesgue measure. Phenomena that are Baire generic, but have a small probability are well known in dynamical systems, KAM theory, number theory, etc. (see [0], [HSY], [K3] for various examples). This partially motivates the problem posed by Arnold $[\mathrm{A}]$

Problem 1. Prove that "with probability one" $f \in \operatorname{Diff}^{r}(M)$ is an A-M diffeomorphism.

Arnold suggested the following interpretation of "with probability one": for $a$ (Baire) generic finite parameter family of diffeomorphisms $\left\{f_{\varepsilon}\right\}$, for Lebesgue almost every $\varepsilon$ we have that $f_{\varepsilon}$ is $A-M$ (cf. [K3] $)$. As Theorem 1.3 shows, a result on the genericity of the set of A-M diffeomorphisms based on (Baire) topology 
is likely to be extremely subtle, if possible at all 1 We use instead a notion of "probability one" based on prevalence [HSY, K3, which is independent of Baire genericity. We also are able to state the result in the form Arnold suggested for generic families using this measure-theoretic notion of genericity.

For a rough understanding of prevalence, consider a Borel measure $\mu$ on a Banach space $V$. We say that a property holds " $\mu$-almost surely for perturbations" if it holds on a Borel set $P \subset V$ such that for all $v \in V$ we have $v+w \in P$ for almost every $w$ with respect to $\mu 2$ Notice that if $V=\mathbb{R}^{k}$ and $\mu$ is Lebesgue measure, then " $\mu$-almost surely for perturbations" is equivalent to "Lebesgue almost everywhere". Moreover, the Fubini-Tonelli Theorem implies that if $\mu$ is any Borel probability measure on $\mathbb{R}^{k}$, then a property that holds $\mu$-almost surely for perturbations must also hold Lebesgue almost everywhere. Based on this observation, we call a property on a Banach space "prevalent" if it holds $\mu$-almost surely for perturbations for some Borel probability measure $\mu$ on $V$, which for technical reasons (cf. [HSY]) we require to have compact support. In order to apply this notion to the Banach manifold $\operatorname{Diff}^{r}(M)$, we must describe how we make perturbations in this space, which we will do in the next section.

Our first main result is a partial solution to Arnold's problem. It says that for a prevalent diffeomorphism $f \in \operatorname{Diff}^{r}(M)$, with $1<r \leq \infty$, and all $\delta>0$ there exists $C=C(\delta)>0$ such that for all $n \in \mathbb{Z}_{+}$,

$$
P_{n}(f) \leq \exp \left(C n^{1+\delta}\right)
$$

The Kupka-Smale theorem (see e.g. [PM]) states that for a generic diffeomorphism all periodic points are hyperbolic and all associated stable and unstable manifolds intersect one another transversally. The paper [K3] shows that the KupkaSmale theorem also holds on a prevalent set. So, the Kupka-Smale theorem, in particular, says that a Baire generic (resp. prevalent) diffeomorphism has only hyperbolic periodic points, but how hyperbolic are the periodic points, as functions of their period, for a Baire generic (resp. prevalent) diffeomorphism $f$ ? This is the second main problem we deal with in this paper.

Recall that a linear operator $L: \mathbb{R}^{N} \rightarrow \mathbb{R}^{N}$ is hyperbolic if it has no eigenvalues on the unit circle $\{|z|=1\} \subset \mathbb{C}$. Denote by $|\cdot|$ the Euclidean norm in $\mathbb{C}^{N}$. Then we define the hyperbolicity of a linear operator $L$ by

$$
\gamma(L)=\inf _{\phi \in[0,1)} \inf _{|v|=1}|L v-\exp (2 \pi i \phi) v| .
$$

We also say that $L$ is $\gamma$-hyperbolic if $\gamma(L) \geq \gamma$. In particular, if $L$ is $\gamma$-hyperbolic, then its eigenvalues $\left\{\lambda_{j}\right\}_{j=1}^{N} \subset \mathbb{C}$ are at least $\gamma$-distant from the unit circle, i.e., $\min _{j}|| \lambda_{j}|-1| \geq \gamma$. The hyperbolicity of a periodic point $x=f^{n}(x)$ of period $n$, denoted by $\gamma_{n}(x, f)$, equals the hyperbolicity of the derivative $d f^{n}(x)$ of $f^{n}$ at points $x$, i.e., $\gamma_{n}(x, f)=\gamma\left(d f^{n}(x)\right)$. Similarly to the number of periodic points $P_{n}(f)$ of period $n$, define

$$
\gamma_{n}(f)=\min _{\left\{x: x=f^{n}(x)\right\}} \gamma_{n}(x, f)
$$

\footnotetext{
1 For example, using techniques from [GST2 and K2] one can prove that for a Baire generic finite-parameter family $\left\{f_{\varepsilon}\right\}$ and a Baire generic parameter value $\varepsilon$ the corresponding diffeomorphism $f_{\varepsilon}$ is not A-M. Unfortunately, how to estimate from below the measure of non-A-M diffeomorphisms in a Baire generic finite-parameter family is so far an unreachable question.

${ }^{2} \mathrm{~A}$ similar notion of prevalence is used in [VK].
} 
The idea of Gromov [G] and Yomdin [Y] for measuring hyperbolicity is that a $\gamma$ hyperbolic point of period $n$ of a $C^{2}$ diffeomorphism $f$ has an $M_{2}^{-2 n} \gamma$-neighborhood (where $M_{2}=\|f\|_{C^{2}}$ ) free from periodic points of the same period 3 One can prove the following slightly more general result.

Proposition 1.1. Let $M$ be a compact manifold of dimension $N$, let $f: M \rightarrow M$ be a $C^{1+\rho}$ diffeomorphism (where $0<\rho \leq 1$ ) that has only hyperbolic periodic points, and let $M_{1+\rho}=\max \left(\|f\|_{C^{1+\rho}}, 2^{1 / \rho}\right)$. Then there is a constant $C=C(M)>0$ such that for each $n \in \mathbb{Z}_{+}$we have

$$
P_{n}(f) \leq C\left(M_{1+\rho}\right)^{n N(1+\rho) / \rho} \gamma_{n}(f)^{-N / \rho} .
$$

Proposition 1.1 implies that a lower estimate on the decay of hyperbolicity $\gamma_{n}(f)$ gives an upper estimate on the growth of the number of periodic points $P_{n}(f)$. Therefore, a natural question is:

Question 2. How quickly can $\gamma_{n}(f)$ decay with $n$ for a "generic" $C^{r}$ diffeomorphism $f$ ?

The existence of a lower bound on the rate of decay of $\gamma_{n}(f)$ for Baire generic $f \in \operatorname{Diff}^{r}(M)$ would imply the existence of an upper bound on the rate of growth of the number of periodic points $P_{n}(f)$, whereas no such bound exists by Theorem 1.3 . Thus again we consider genericity in the measure-theoretic sense of prevalence. Our second main result, which in view of Proposition 1.1 implies the first main result, is that for a prevalent diffeomorphism $f \in \operatorname{Diff}^{r}(M)$, with $1<r \leq \infty$, and all $\delta>0$ there exists $C=C(\delta)>0$ such that

$$
\gamma_{n}(f) \geq \exp \left(-C n^{1+\delta}\right) .
$$

Now we shall discuss in more detail our definition of prevalence ("probability one") in the space of diffeomorphisms $\operatorname{Diff}^{r}(M)$.

\section{Prevalence in the space of Diffeomorphisms $\operatorname{Diff}^{r}(M)$}

The space of $C^{r}$ diffeomorphisms $\operatorname{Diff}^{r}(M)$ of a compact manifold $M$ is a Banach manifold. Locally we can identify it with a Banach space, which gives it a local linear structure in the sense that we can perturb a diffeomorphism by "adding" small elements of the Banach space. As we described in the previous section, the notion of prevalence requires additive perturbations with respect to a probability measure that is independent of the place that the perturbation is performed. Thus although there is not a unique way to put a linear structure on $\operatorname{Diff}^{r}(M)$, it is important to make a choice that is consistent throughout the Banach manifold.

The way we make perturbations on $\operatorname{Diff}^{r}(M)$ by small elements of a Banach space is as follows. First we embed $M$ into the interior of the closed unit ball $B^{N} \subset \mathbb{R}^{N}$, which we can do for $N$ sufficiently large by the Whitney Embedding Theorem $[\mathrm{W}$. We emphasize that our results hold for every possible choice of an embedding of $M$ into $\mathbb{R}^{N}$. We then consider a closed tubular neighborhood $U \subset B^{N}$ of $M$ and the Banach space $C^{r}\left(U, \mathbb{R}^{N}\right)$ of $C^{r}$ functions from $U$ to $\mathbb{R}^{N}$. Next, we extend every element $f \in \operatorname{Diff}^{r}(M)$ to an element $F \in C^{r}\left(U, \mathbb{R}^{N}\right)$ that is strongly contracting in the directions transverse to $M$. Again the particular choice

\footnotetext{
${ }^{3}$ In $[Y]$ hyperbolicity is introduced as the minimal distance of eigenvalues to the unit circle. This way of defining hyperbolicity does not guarantee the existence of a $M_{2}^{-2 n} \gamma$-neighborhood free from periodic points of the same period $[\mathrm{KH}]$.
} 
of how we make this extension is not important to our results; in the Appendix we describe one way to make this extension so that the results of Sacker $\mathrm{Sac}$ and Fenichel $[\mathrm{F}]$ apply as follows. Since $F$ has $M$ as an invariant manifold, if we add to $F$ a small perturbation in $g \in C^{r}\left(U, \mathbb{R}^{N}\right)$, the perturbed map $F+g$ has an invariant manifold in $U$ that is close to $M$. Then $F+g$ restricted to its invariant manifold corresponds in a natural way to an element of $\operatorname{Diff}^{r}(M)$, which we consider to be the perturbation of $f \in \operatorname{Diff}^{r}(M)$ by $g \in C^{r}\left(U, \mathbb{R}^{N}\right)$. The details of this construction are described in the Appendix.

In this way we reduce the problem to the study of maps in $\operatorname{Diff}^{r}(U)$, the open subset of $C^{r}\left(U, \mathbb{R}^{N}\right)$ consisting of those elements that are diffeomorphisms from $U$ to some subset of its interior. The construction we described in the previous paragraph ensures that the number of periodic points $P_{n}(f)$ and their hyperbolicity $\gamma_{n}(f)$ for elements of $\operatorname{Diff}^{r}(M)$ are the same for the corresponding elements of $\operatorname{Diff}^{r}(U)$, so the bounds that we prove on these quantities for almost every perturbation of any element of $\operatorname{Diff}^{r}(U)$ hold as well for almost every perturbation of any element of $\operatorname{Diff}^{r}(M)$. Another justification for considering diffeomorphisms in Euclidean space is that the problem of exponential/superexponential growth of the number of periodic points $P_{n}(f)$ for a prevalent $f \in \operatorname{Diff}^{r}(M)$ is a local problem on $M$ and is not affected by a global shape of $M$.

The results stated in the next section apply to any compact domain $U \subset \mathbb{R}^{N}$, but for simplicity we state them for the closed unit ball $B^{N}$. In the previous section, we said that a property is prevalent on a Banach space such as $C^{r}\left(B^{N}, \mathbb{R}^{N}\right)$ if it holds on a Borel subset $S$ for which there exists a Borel probability measure $\mu$ on $C^{r}\left(B^{N}, \mathbb{R}^{N}\right)$ with compact support such that for all $F \in C^{r}\left(B^{N}, \mathbb{R}^{N}\right)$ we have $F+g \in S$ for almost every $g$ with respect to $\mu$. The complement of a prevalent set is said to be shy. We then say that a property is prevalent on an open subset of $C^{r}\left(B^{N}, \mathbb{R}^{N}\right)$ such as $\operatorname{Diff}^{r}\left(B^{N}\right)$ if the exceptions to the property in $\operatorname{Diff}^{r}\left(B^{N}\right)$ form a shy subset of $C^{r}\left(B^{N}, \mathbb{R}^{N}\right)$.

In this paper the perturbation measure $\mu$ that we use is supported within the analytic functions in $C^{r}\left(B^{N}, \mathbb{R}^{N}\right)$. In this sense we foliate $\operatorname{Diff}^{r}\left(B^{N}\right)$ by analytic leaves that are compact and overlapping. The main result then says that for every analytic leaf $L \subset \operatorname{Diff}^{r}\left(B^{N}\right)$ and every $\delta>0$, for almost every diffeomorphism $f \in L$ in the leaf $L$ both (5) and (9) are satisfied. Now we define an analytic leaf as a "Hilbert brick" in the space of analytic functions, and a natural product Lebesgue probability measure $\mu$ on it.

\section{Formulation of MAin Results}

Fix a coordinate system $x=\left(x_{1}, \ldots, x_{N}\right) \in \mathbb{R}^{N} \supset B^{N}$ and the scalar product $\langle x, y\rangle=\sum_{i} x_{i} y_{i}$. Let $\alpha=\left(\alpha_{1}, \ldots, \alpha_{N}\right)$ be a multiindex from $\mathbb{Z}_{+}^{N}$, and let $|\alpha|=$ $\sum_{i} \alpha_{i}$. For a point $x=\left(x_{1}, \ldots, x_{N}\right) \in \mathbb{R}^{N}$ we write $x^{\alpha}=\prod_{i=1}^{N} x_{i}^{\alpha_{i}}$. Associate to a real analytic function $\phi: B^{N} \rightarrow \mathbb{R}^{N}$ the set of coefficients of its expansion:

$$
\phi_{\vec{\varepsilon}}(x)=\sum_{\alpha \in \mathbb{Z}_{+}^{N}} \vec{\varepsilon}_{\alpha} x^{\alpha} .
$$

Denote by $W_{k, N}$ the space of $N$-component homogeneous vector-polynomials of degree $k$ in $N$ variables, and by $\nu(k, N)=\operatorname{dim} W_{k, N}$ the dimension of $W_{k, N}$. According to the notation of the expansion (10), denote coordinates in $W_{k, N}$ by

$$
\vec{\varepsilon}_{k}=\left(\left\{\vec{\varepsilon}_{\alpha}\right\}_{|\alpha|=k}\right) \in W_{k, N} .
$$


In $W_{k, N}$ we use a scalar product that is invariant with respect to the orthogonal transformation of $\mathbb{R}^{N} \supset B^{N}$ defined as follows:

$$
\left\langle\vec{\varepsilon}_{k}, \vec{\zeta}_{k}\right\rangle_{k}=\sum_{|\alpha|=k}\left(\begin{array}{c}
k \\
\alpha
\end{array}\right)^{-1}\left\langle\vec{\varepsilon}_{\alpha}, \vec{\zeta}_{\alpha}\right\rangle, \quad\left\|\vec{\varepsilon}_{k}\right\|_{k}=\left(\left\langle\vec{\varepsilon}_{k}, \vec{\varepsilon}_{k}\right\rangle_{k}\right)^{1 / 2} .
$$

Denote by

$$
B_{k}^{N}(r)=\left\{\vec{\varepsilon}_{k} \in W_{k, N}:\left\|\vec{\varepsilon}_{k}\right\|_{k} \leq r\right\}
$$

the closed $r$-ball in $W_{k, N}$ centered at the origin. Let $L e b_{k, N}$ be Lebesgue measure on $W_{k, N}$ induced by the scalar product (12) and normalized by a constant so that the volume of the unit ball is one: $\operatorname{Leb}_{k, N}\left(B_{k}^{N}(1)\right)=1$.

Fix a nonincreasing sequence of positive numbers $\mathbf{r}=\left(\left\{r_{k}\right\}_{k=0}^{\infty}\right)$ such that $r_{k} \rightarrow 0$ as $k \rightarrow \infty$ and define a Hilbert brick of size $\mathbf{r}$,

$$
\begin{aligned}
H B^{N}(\mathbf{r}) & =\left\{\vec{\varepsilon}=\left\{\vec{\varepsilon}_{\alpha}\right\}_{\alpha \in \mathbb{Z}_{+}^{N}}: \text { for all } k \in \mathbb{Z}_{+},\left\|\vec{\varepsilon}_{k}\right\|_{k} \leq r_{k}\right\} \\
& =B_{0}^{N}\left(r_{0}\right) \times B_{1}^{N}\left(r_{1}\right) \times \cdots \times B_{k}^{N}\left(r_{k}\right) \times \cdots \\
& \subset W_{0, N} \times W_{1, N} \times \cdots \times W_{k, N} \times \cdots .
\end{aligned}
$$

Define a product Lebesgue probability measure $\mu_{\mathbf{r}}^{N}$ associated to the Hilbert brick $H B^{N}(\mathbf{r})$ of size $\mathbf{r}$ by normalizing for each $k \in \mathbb{Z}_{+}$the corresponding Lebesgue measure $L e b_{k, N}$ on $W_{k, N}$ to the Lebesgue probability measure on the $r_{k}$-ball $B_{k}^{N}\left(r_{k}\right)$ :

$$
\mu_{k, r}^{N}=r^{-\nu(k, N)} L e b_{k, N} \quad \text { and } \quad \mu_{\mathbf{r}}^{N}=\chi_{k=0}^{\infty} \mu_{k, r_{k}}^{N} .
$$

Definition 3.1. Let $f \in \operatorname{Diff}^{r}\left(B^{N}\right)$ be a $C^{r}$ diffeomorphism of $B^{N}$ into its interior. We call $H B^{N}(\mathbf{r})$ a Hilbert brick of an admissible size $\mathbf{r}=\left(\left\{r_{k}\right\}_{k=0}^{\infty}\right)$ with respect to $f$ if:

A) for each $\vec{\varepsilon} \in H B^{N}(\mathbf{r})$, the corresponding function $\phi_{\vec{\varepsilon}}(x)=\sum_{\alpha \in \mathbb{Z}_{+}^{N}} \vec{\varepsilon}_{\alpha} x^{\alpha}$ is analytic on $B^{N}$;

B) for each $\vec{\varepsilon} \in H B^{N}(\mathbf{r})$, the corresponding map $f_{\vec{\varepsilon}}(x)=f(x)+\phi_{\vec{\varepsilon}}(x)$ is a diffeomorphism from $B^{N}$ into its interior, i.e., $\left\{f_{\vec{\varepsilon}}\right\}_{\vec{\varepsilon} \in H B^{N}(\mathbf{r})} \subset \operatorname{Diff}^{r}\left(B^{N}\right)$;

C) for all $\delta>0$ and all $C>0$, the sequence $r_{k} \exp \left(C k^{1+\delta}\right) \rightarrow \infty$ as $k \rightarrow \infty$.

Remark 3.1. The first and second conditions ensure that the family $\left\{f_{\vec{\varepsilon}}\right\}_{\vec{\varepsilon} \in H B^{N}(\mathbf{r})}$ lie in an analytic leaf within the class of diffeomorphisms $\operatorname{Diff}^{r}\left(B^{N}\right)$. The third condition provides us enough freedom to perturb. It is important for our method to have infinitely many parameters to perturb. If the $r_{k}$ decayed too fast to zero, it would make our family of perturbations essentially finite-dimensional.

An example of an admissible sequence $\mathbf{r}=\left(\left\{r_{k}\right\}_{k=0}^{\infty}\right)$ is $r_{k}=\tau / k$ !, where $\tau$ depends on $f$ and is chosen sufficiently small to ensure that condition (B) holds. Notice that the diameter of $H B^{N}(\mathbf{r})$ is then proportional to $\tau$, so that $\tau$ can be chosen as some multiple of the distance from $f$ to the boundary of $\operatorname{Diff}^{r}\left(B^{N}\right)$.

Main Theorem. For each $0<\rho \leq \infty$ and every $C^{1+\rho}$ diffeomorphism $f \in$ $\operatorname{Diff}^{1+\rho}\left(B^{N}\right)$, consider a Hilbert brick $H B^{N}(\mathbf{r})$ of an admissible size $\mathbf{r}$ with respect to $f$ and the family of analytic perturbations of $f$,

$$
\left\{f_{\vec{\varepsilon}}(x)=f(x)+\phi_{\vec{\varepsilon}}(x)\right\}_{\vec{\varepsilon} \in H B^{N}(\mathbf{r})}
$$


with the product Lebesgue probability measure $\mu_{\mathbf{r}}^{N}$ associated to $H B^{N}(\mathbf{r})$. Then for every $\delta>0$ and for $\mu_{\mathbf{r}}^{N}$-a.e. $\vec{\varepsilon}$ there is $C=C(\vec{\varepsilon}, \delta)>0$ such that for all $n \in \mathbb{Z}_{+}$

$$
\gamma_{n}\left(f_{\vec{\varepsilon}}\right)>\exp \left(-C n^{1+\delta}\right), \quad P_{n}\left(f_{\vec{\varepsilon}}\right)<\exp \left(C n^{1+\delta}\right) .
$$

Remark 3.2. The fact that the measure $\mu_{\mathbf{r}}^{N}$ depends on $f$ does not conform to our definition of prevalence. However, we can decompose $\operatorname{Diff}^{r}\left(B^{N}\right)$ into a nested countable union of sets $\mathcal{S}_{j}$ that are each a positive distance from the boundary of $\operatorname{Diff}^{r}\left(B^{N}\right)$ and for each $j \in \mathbb{Z}^{+}$choose an admissible sequence $\mathbf{r}_{j}$ that is valid for all $f \in \mathcal{S}_{j}$. Since a countable intersection of prevalent subsets of a Banach space is prevalent [HSY], the Main Theorem implies the results stated in terms of prevalence in the Introduction.

In the Appendix we deduce from the Main Theorem the following result.

Theorem 3.1. Let $\left\{f_{\varepsilon}\right\}_{\varepsilon \in B^{m}} \subset \operatorname{Diff}^{1+\rho}(M)$ be a generic m-parameter family of $C^{1+\rho}$ diffeomorphisms of a compact manifold $M$ for some $\rho>0$. Then for every $\delta>0$ and a.e. $\varepsilon \in B^{m}$ there is a constant $C=C(\varepsilon, \delta)$ such that (17) is satisfied for every $n \in \mathbb{Z}_{+}$.

In the Appendix we also give a precise meaning to the term generic.

Let us formulate the most general result we shall prove.

Definition 3.2. Let $\gamma \geq 0$ and $f \in \operatorname{Diff}^{1+\rho}\left(B^{N}\right)$ be a $C^{1+\rho}$ diffeomorphism for some $\rho>0$. A point $x \in B^{N}$ is called $(n, \gamma)$-periodic if $\left\|f^{n}(x)-x\right\| \leq \gamma$, and $(n, \gamma)$-hyperbolic if $\gamma_{n}(x, f)=\gamma\left(d f^{n}(x)\right) \geq \gamma$.

(Notice that a point can be $(n, \gamma)$-hyperbolic regardless of its periodicity, but this property is of interest primarily for $(n, \gamma)$-periodic points.) For positive $C$ and $\delta$ let $\gamma_{n}(C, \delta)=\exp \left(-C n^{1+\delta}\right)$.

Theorem 3.2. Given the hypotheses of the Main Theorem, for every $\delta>0$ and for $\mu_{\mathbf{r}}^{N}$-a.e. $\vec{\varepsilon}$ there is $C=C(\vec{\varepsilon}, \delta)>0$ such that for all $n \in \mathbb{Z}_{+}$, every $\left(n, \gamma_{n}^{1 / \rho}(C, \delta)\right)$ periodic point $x \in B^{N}$ is $\left(n, \gamma_{n}(C, \delta)\right)$-hyperbolic. (Here we assume $0<\rho \leq 1$; in a space Diff ${ }^{1+\rho}\left(B^{N}\right)$ with $\rho>1$, the statement holds with $\rho$ replaced by 1.$)$

This result together with Proposition 1.1 implies the Main Theorem, because every periodic point of period $n$ is $(n, \gamma)$-periodic for all $\gamma>0$.

Remark 3.3. In the statement of the Main Theorem and Theorem 3.2 the unit ball $B^{N}$ can be replaced by a bounded open set $U \subset \mathbb{R}^{N}$. After scaling, $U$ can be regarded as a subset of the unit ball $B^{N}$.

One can define a distance on a compact manifold $M$ and almost periodic points of diffeomorphisms of $M$. Then one can cover $M=\bigcup_{i} U_{i}$ by coordinate charts and define hyperbolicity for almost periodic points using these charts $\left\{U_{i}\right\}_{i}$ (see $[\mathrm{Y}]$ for details). This gives a precise meaning to the following result.

Theorem 3.3. Let $\left\{f_{\varepsilon}\right\}_{\varepsilon \in B^{m}} \subset \operatorname{Diff}^{1+\rho}(M)$ be a generic m-parameter family of diffeomorphisms of a compact manifold $M$ for some $\rho>0$. Then for every $\delta>$ 0 and almost every $\varepsilon \in B^{m}$ there is a constant $C=C(\varepsilon, \delta)$ such that every $\left(n, \gamma_{n}^{1 / \rho}(C, \delta)\right)$-periodic point $x$ in $B^{N}$ is $\left(n, \gamma_{n}(C, \delta)\right)$-hyperbolic. (Here again we assume $0<\rho \leq 1$, replacing $\rho$ with 1 in the conclusion if $\rho>1$.)

The meaning of the term generic is the same as in Theorem 3.1 and is discussed in the Appendix. 


\section{Formulation of the main Result in the 1-Dimensional CASE}

The proof of the main result about estimating the rate of growth of the number of periodic points for diffeomorphisms in $N$ dimensions has many complications related to multidimensionality. To describe a model which is, on one hand, nontrivial, and on the other hand, useful for understanding the general technique, we apply our method to the 1-dimensional maps. The statement of the main result for the 1-dimensional maps has another important feature: it explains the statement of the main multidimensional result.

Fix the interval $I=[-1,1]$. Associate to a real analytic function $\phi: I \rightarrow \mathbb{R}$ the set of coefficients of its expansion

$$
\phi_{\varepsilon}(x)=\sum_{k=0}^{\infty} \varepsilon_{k} x^{k} .
$$

For a nonincreasing sequence of positive numbers $\mathbf{r}=\left(\left\{r_{k}\right\}_{k=0}^{\infty}\right)$ such that $r_{k} \rightarrow 0$ as $k \rightarrow \infty$, following the multidimensional notation, we define a Hilbert brick of size $\mathbf{r}$,

$$
H B^{1}(\mathbf{r})=\left\{\varepsilon=\left\{\varepsilon_{k}\right\}_{k=0}^{\infty}: \text { for all } k \in \mathbb{Z}_{+},\left|\varepsilon_{k}\right| \leq r_{k}\right\},
$$

and the product probability measure $\mu_{\mathbf{r}}^{1}$ associated to the Hilbert brick $H B^{1}(\mathbf{r})$ of size $\mathbf{r}$, which considers each $\varepsilon_{k}$ to be an independent random variable uniformly distributed on $\left[-r_{k}, r_{k}\right]$.

Main 1-dimensional Theorem. For each $0<\rho \leq \infty$ and every $C^{1+\rho}$ map $f$ : $I \rightarrow I$ of the interval $I=[-1,1]$ consider a Hilbert brick $H B^{1}(\mathbf{r})$ of an admissible size $\mathbf{r}$ with respect to $f$ and the family of analytic perturbations of $f$,

$$
\left\{f_{\varepsilon}(x)=f(x)+\phi_{\varepsilon}(x)\right\}_{\varepsilon \in H B^{1}(\mathbf{r})},
$$

with the product Lebesgue probability measure $\mu_{\mathbf{r}}^{1}$ associated to $H B^{1}(\mathbf{r})$. Then for every $\delta>0$ and $\mu_{\mathbf{r}}^{1}$-a.e. $\varepsilon$ there is $C=C(\varepsilon, \delta)>0$ such that for all $n \in \mathbb{Z}_{+}$

$$
\gamma_{n}\left(f_{\varepsilon}\right)>\exp \left(-C n^{1+\delta}\right), \quad P_{n}\left(f_{\varepsilon}\right)<\exp \left(C n^{1+\delta}\right) .
$$

In [MMS Martens-de Melo-Van Strien prove in a sense a stronger statement for $C^{2}$ maps. They showed that for any $C^{2}$ map $f$ of an interval without "flat" critical points there are some $\gamma>0$ and $n_{0} \in \mathbb{Z}_{+}$such that for any $n>n_{0}$ we have $\gamma_{n}(f)>1+\gamma$. This also implies that the number of periodic points is bounded by an exponential function of the period. The notion of a flat critical point used in [MMS] is a nonstandard one from the point of view of singularity theory. For $C^{2}$ maps, the authors call $x_{0}$ a flat critical point of $f$ if $f^{\prime}\left(x_{0}\right)=f^{\prime \prime}\left(x_{0}\right)=0$; the distance from $f(x)$ to $f\left(x_{0}\right)$ does not have to decay to 0 as $x \rightarrow x_{0}$ faster than any power of $x-x_{0}$.

In $\left[\mathrm{KK}\right.$ an example of a $C^{2}$-unimodal map with a critical point having tangency of order 4 and an arbitrarily fast rate of growth of the number of periodic points is given. Another advantage of the Main 1-dimensional Theorem is that it works for $C^{1+\rho}$ maps with $0<\rho<1$, whereas the result in [MMS] works only for $C^{2}$ maps.

\section{Strategy of the Proof}

Here we describe the strategy of the proof of Theorem 3.2 . The basic technique is developed and many of the technical difficulties are resolved by the first author in [K4]. The general idea is to fix $C>0$ and prove an upper bound on the measure 
of the set of "bad" parameter values $\vec{\varepsilon} \in H B^{N}(\mathbf{r})$ for which the conclusion of the theorem does not hold. The upper bound we obtain will approach zero as $C \rightarrow \infty$, from which it follows immediately that the set of $\vec{\varepsilon} \in H B^{N}(\mathbf{r})$ that are "bad" for all $C>0$ has measure zero. For a given $C>0$, we bound the measure of "bad" parameter values inductively as follows.

Stage 1. We delete all parameter values $\vec{\varepsilon} \in H B^{N}(\mathbf{r})$ for which the corresponding diffeomorphism $f_{\vec{\varepsilon}}$ has an almost fixed point that is not sufficiently hyperbolic, and bound the measure of the deleted set.

Stage 2. After Stage 1, we consider only parameter values for which all almost fixed points are sufficiently hyperbolic. Then we delete all parameter values $\vec{\varepsilon}$ for which $f_{\vec{\varepsilon}}$ has an almost periodic point of period 2 which is not sufficiently hyperbolic, and bound the measure of that set.

Stage $n$. We consider only parameter values for which all almost periodic points of period at most $n-1$ are sufficiently hyperbolic (we shall call this the Inductive Hypothesis). Then we delete all parameter values $\vec{\varepsilon}$ for which $f_{\vec{\varepsilon}}$ has an almost periodic point of period $n$ which is not sufficiently hyperbolic, and bound the measure of that set.

The main difficulty in the proof is then to find a bound on the measure of "bad" parameter values at stage $n$ such that the bounds are summable over $n$ and that the sum approaches zero as $C \rightarrow \infty$. Let us formalize the problem. Fix positive $\rho$, $\delta$, and $C$, and recall that $\gamma_{n}(C, \delta)=\exp \left(-C n^{1+\delta}\right)$ for $n \in \mathbb{Z}_{+}$. Assume $\rho \leq 1$; if not, change its value to 1 .

Definition 5.1. A diffeomorphism $f \in \operatorname{Diff}^{1+\rho}\left(B^{N}\right)$ satisfies the Inductive Hypothesis of order $n$ with constants $(C, \delta, \rho)$, denoted $f \in I H(n, C, \delta, \rho)$, if for all $k \leq n$, every $\left(k, \gamma_{k}^{1 / \rho}(C, \delta)\right)$-periodic point is $\left(k, \gamma_{k}(C, \delta)\right)$-hyperbolic.

For $f \in \operatorname{Diff}^{1+\rho}(M)$, consider the sequence of sets

$$
B_{n}(C, \delta, \rho, \mathbf{r}, f)=\left\{\vec{\varepsilon} \in H B^{N}(\mathbf{r}): f_{\vec{\varepsilon}} \in I H(n-1, C, \delta, \rho) \text { but } f_{\vec{\varepsilon}} \notin I H(n, C, \delta, \rho)\right\}
$$

in the parameter space $H B^{N}(\mathbf{r})$. In other words, $B_{n}(C, \delta, \rho, \mathbf{r}, f)$ is the set of "bad" parameter values $\vec{\varepsilon} \in H B^{N}(\mathbf{r})$ for which all almost periodic points of $f_{\vec{\varepsilon}}$ with period strictly less than $n$ are sufficiently hyperbolic, but there is an almost periodic point of period $n$ that is not sufficiently hyperbolic. Let

$$
\begin{aligned}
M_{1} & =\sup _{\vec{\varepsilon} \in H B^{N}(\mathbf{r})} \max \left\{\left\|f_{\vec{\varepsilon}}\right\|_{C^{1}},\left\|f_{\vec{\varepsilon}}^{-1}\right\|_{C^{1}}\right\} ; \\
M_{1+\rho} & =\sup _{\vec{\varepsilon} \in H B^{N}(\mathbf{r})} \max \left\{\left\|f_{\vec{\varepsilon}}\right\|_{C^{1+\rho}}, M_{1}, 2^{1 / \rho}\right\} .
\end{aligned}
$$

Our goal is to find an upper bound $\mu_{n}\left(C, \delta, \rho, \mathbf{r}, M_{1+\rho}\right)$ for the measure

$$
\mu_{\mathbf{r}}^{N}\left(B_{n}(C, \delta, \rho, \mathbf{r}, f)\right)
$$

of the set of "bad" parameter values. Then $\sum_{n=1}^{\infty} \mu_{n}\left(C, \delta, \rho, \mathbf{r}, M_{1+\rho}\right)$ is an upper bound for the measure of $\bigcup_{n=1}^{\infty} B_{n}(C, \delta, \rho, \mathbf{r}, f)$, which is the set of all parameter values $\vec{\varepsilon}$ for which $f_{\vec{\varepsilon}}$ has (for some $\left.n\right)$ an $\left(n, \gamma_{n}^{1 / \rho}(C, \delta)\right)$-periodic point that is not $\left(n, \gamma_{n}(C, \delta)\right)$-hyperbolic. If this sum converges and

$$
\sum_{n=1}^{\infty} \mu_{n}\left(C, \delta, \rho, \mathbf{r}, M_{1+\rho}\right)=\mu\left(C, \delta, \rho, \mathbf{r}, M_{1+\rho}\right) \rightarrow 0 \quad \text { as } C \rightarrow \infty
$$


for every positive $\rho, \delta$, and $M_{1+\rho}$, then Theorem 3.2 follows. In the subsequent article we describe the key construction we use to obtain a bound $\mu_{n}\left(C, \delta, \rho, \mathbf{r}, M_{1+\rho}\right)$ that meets condition (24).

\section{Appendix: Extension of $\operatorname{Diff}^{r}(M)$ to Euclidean space}

Here we explain how to extend a diffeomorphism $f \in \operatorname{Diff}^{r}(M)$ to a smooth function on a tubular neighborhood of $M$, as described in Section 2. Given a smooth $\left(C^{\infty}\right)$ compact manifold $M$ of dimension $D$, for $N>2 D$ the Whitney Embedding Theorem says that a generic smooth function from $M$ to $\mathbb{R}^{N}$ is a diffeomorphism between $M$ and its image. To simplify notation, we identify $M$ with its image, so that $M$ becomes a submanifold of $\mathbb{R}^{N}$.

Let $U \subset \mathbb{R}^{N}$ be a closed neighborhood of $M$, chosen sufficiently small that there is a well-defined projection $\pi: U \rightarrow M$ for which $\pi(x)$ is the closest point in $M$ to $x$. Then for each $y \in M, \pi^{-1}(y)$ is an $(N-D)$-dimensional disk. We then extend each $f \in \operatorname{Diff}^{r}(M)$ to a function $F \in C^{r}(U)$ that is a diffeomorphism from $U$ to a subset of its interior as follows. For each $0<\rho<1$ and $y \in M$ choose a linear function $h_{\rho, y, f}: \pi^{-1}(y) \rightarrow \pi^{-1}(f(y))$ that maps $y$ to $f(y)$ and contracts distances by a factor of $\rho$, and such that $h_{\rho, y, f}$ depends continuously on $f$ and is $C^{r}$ as a function of $y$. Let $\rho=\min \left(\left\|f^{-1}\right\|_{C^{1}}^{-r}, 1\right) / 2$ and let

$$
F(x)=h_{\rho, \pi(x), f}(x) .
$$

Then by Fenichel's Theorem [F], every sufficiently small perturbation $F_{\varepsilon} \in C^{r}(U)$ of such an $F$ has an invariant manifold $M_{\varepsilon} \subset U$ for which $\left.\pi\right|_{M_{\varepsilon}}$ is a $C^{r}$ diffeomorphism from $M_{\varepsilon}$ to $M$. Then to such an $F_{\varepsilon}$ we can associate a diffeomorphism $f_{\varepsilon} \in$ $\operatorname{Diff}^{r}(M)$ by letting

$$
f_{\varepsilon}(y)=\pi\left(F_{\varepsilon}\left(\left.\pi\right|_{M_{\varepsilon}} ^{-1}(y)\right)\right) .
$$

Notice that the periodic points of $F_{\varepsilon}$ all lie on $M_{\varepsilon}$ and are in one-to-one correspondence with the periodic points of $f_{\varepsilon}$. Furthermore, because $f_{\varepsilon}$ and $\left.F_{\varepsilon}\right|_{M_{\varepsilon}}$ are conjugate, the hyperbolicity of each periodic orbit is the same for either map. Thus any estimate of $P_{n}\left(F_{\varepsilon}\right)$ or $\gamma_{n}\left(F_{\varepsilon}\right)$ applies also to $f_{\varepsilon}$.

\section{REFERENCES}

[A] Problems of Arnold's seminar, FAZIS, Moscow, 2000.

[AM] M. Artin and B. Mazur, On periodic points, Ann. Math. 81 (1965), 82-99. MR 31:754

[F] N. Fenichel, Persistence and smoothness of invariant manifolds for flows, Indiana Univ. Math. J. 21 (1971), 193-226. MR 44:4313

[GST1] S. V. Gonchenko, L. P. Shil'nikov, D. V. Turaev, On models with non-rough Poincaré homoclinic curves, Physica D 62 (1993), 1-14. MR 94c:58098

[GST2] S. Gonchenko, L. Shil'nikov, D. Turaev, Homoclinic tangencies of an arbitrary order in Newhouse regions, Preprint, in Russian.

[G] M. Gromov, On entropy of holomorphic maps, Preprint.

[HSY] B. R. Hunt, T. Sauer, J. A. Yorke, Prevalence: a translation-invariant "almost every" for infinite-dimensional spaces, Bull. Amer. Math. Soc. 27 (1992), 217-238; Prevalence: an addendum, Bull. Amer. Math. Soc. 28 (1993), 306-307. MR 93k:28018, MR 93k:28019

[K1] V. Yu. Kaloshin, An extension of the Artin-Mazur theorem, Ann. Math. 150 (1999), 729-741. MR 2000j:37020

[K2] V. Yu. Kaloshin, Generic diffeomorphisms with superexponential growth of number of periodic orbits, Comm. Math. Phys. 211 (2000), no.1, 253-271. CMP 2000:12

[K3] V. Yu. Kaloshin, Some prevalent properties of smooth dynamical systems, Tr. Mat. Inst. Steklova 213 (1997), 123-151. MR 99h:58100

[K4] V. Yu. Kaloshin, Ph.D. thesis, Princeton University, 2001. 
$[\mathrm{KH}] \quad$ V. Kaloshin, B. Hunt, Stretched exponential bound on growth of the number of periodic points for prevalent diffeomorphisms, part 2, in preparation.

[KK] V. Kaloshin, O. Kozlovski, An example of a $C^{2}$-unimodal map with an arbitrarily fast growth of the number of periodic points, in preparation

[MMS] M. Martens, W. de Melo, S. Van Strien, Julia-Fatou-Sullivan theory for real onedimensional dynamics, Acta Math. 168 (1992), no. 3-4, 273-318. MR 93d:58137

[PM] J. Palis and W. de Melo, Geometric Theory of Dynamical Systems: An Introduction, Springer-Verlag, 1982. MR 84a:58004

[O] J. C. Oxtoby, Measure and Category, Springer-Verlag, 1971. MR 52:14213

[Sac] R. J. Sacker, A perturbation theorem for invariant manifolds and Hölder continuity, J. Math. Mech. 18 (1969), 705-762. MR 39:578

[VK] M. Vishik, S. Kuksin, Quasilinear elliptic equations and Fredholm manifolds, Moscow Univ. Math. Bull. 40 (1985), no. 6, 26-34. MR 88a:35086

[W] H. Whitney, Differentiable manifolds, Ann. Math. 37 (1936), 645-680.

[Y] Y. Yomdin, A quantitative version of the Kupka-Smale Theorem, Ergod. Th. Dynam. Sys. 5 (1985), 449-472. MR 87c:58091

Fine Hall, Princeton University, Princeton, NJ 08544

E-mail address: kaloshin@math.princeton.edu

Department of Mathematics and Institute for Physical Science and Technology, University of Maryland, College Park, MD 20742

E-mail address: bhunt@ipst.umd.edu 